\title{
Application of Macrofiber Composite for Smart Transducer of Lamb Wave Inspection
}

\author{
Gang Ren and Kyung-Young Jhang \\ School of Mechanical Engineering, Hanyang University, Seoul 133-791, Republic of Korea
}

Correspondence should be addressed to Kyung-Young Jhang; kyjhang@hanyang.ac.kr

Received 4 October 2013; Accepted 20 October 2013

Academic Editor: Kean Aw

Copyright (C) 2013 G. Ren and K.-Y. Jhang. This is an open access article distributed under the Creative Commons Attribution License, which permits unrestricted use, distribution, and reproduction in any medium, provided the original work is properly cited.

Macrofiber composite (MFC) has been developed recently as a new type of smart material for piezoelectric transducers. It shows advantages over traditional piezoelectric ceramic materials (PZT) including the method of application, sensitivity, and cost. It can be embedded on the structure, which provides the possibility to monitor the structural health in real time. In this paper, the feasibility of this transducer for the Lamb wave inspection has been experimentally explored. A pair of MFC patches is bonded on a $2 \mathrm{~mm}$ thick aluminum plate, and it has been demonstrated that the dispersive characteristics of S0 and A0 modes, generated and detected by MFC patches, agreed well with the theory. The influence of the bonding condition of the transducer was also tested to show that rigid bonding is required to assure a high amplitude signal. In order to illustrate the performance of defect detection, an artificial defect fabricated on the surface of a specimen was inspected in the pitch-catch mode. The results showed that the MFC transducer is a promising Lamb wave transducer for nondestructive testing (NDT) and structural health monitoring (SHM).

\section{Introduction}

With a view to enhance the safety and reliability in nondestructive evaluation (NDE), the development of highly efficient techniques for nondestructive damage detection or structural health monitoring is of vital significance [1]. Such SHM requires small, lightweight, cheap, and sensitive smart transducers to be embedded on the surface of the structure at affordable cost, especially in the aerospace field. In addition, the traditional PZT is not suitable for this situation because of limitations such as being monolithic, inflexible, and brittle.

In order to make up for these limitations, a series of smart material transducers was developed in the past two decades. Piezoelectric films (PVDFs) have been used in SHM system [2-4]. Compared to the traditional PZT transducers, they have some advantages of durability and flexibility, but due to poor electromechanical coupling efficiency, they need greater actuation power in generation and heavy amplification in detection [5]. There are other transducers, including active fiber composites (AFCs) and macrofiber composites (MFCs) developed at NASA Langley Research Center, and these transducers avoid some of the limitations of PVDFs. AFCs and MFCs are composed of thin piezoceramic fibers sandwiched between layers of adhesive, electrodes, and a polyimide film [6]. These types of transducers produce higher force and strain than the typical monolithic piezoceramic materials $[7,8]$. The main advantage of MFCs over AFCs is the reduced manufacturing cost, owing to the fibers being sliced into rectangular shapes for MFCs, while the circular AFC fibers are manufactured through a very costly extrusion process [9].

Thus, the developed MFC patch is a promising alternative to the traditional brittle PZT. Also, it is appealing for SHM due to its advantages of flexibility, durability, sensitivity, and reliability. Many researchers have engaged in applying this kind of composite transducer to the nondestructive evaluation field, such as welding defect detection, pipeline inspection, and structural health monitoring $[1,3,10,11]$.

In this paper, we focused on the feasibility of using MFC patches for the Lamb wave inspection. For this, a pair of MFC patches was bonded onto a $2 \mathrm{~mm}$ thick aluminum plate, and the Lamb wave was transmitted and received. The influence 


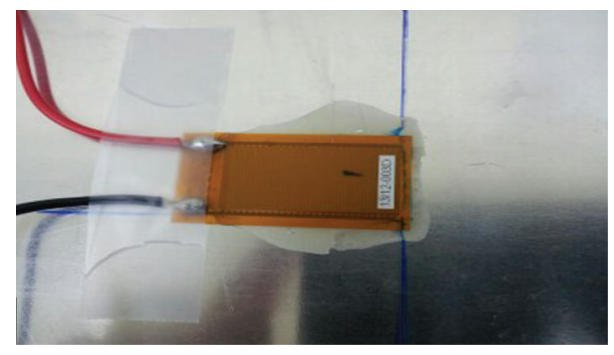

Figure 1: MFC Type P1 M2814 (made by Smart Material Corp.).

TABLE 1: Properties of MFC Type P1 M2814.

\begin{tabular}{lc}
\hline Operational voltage & $-500 \mathrm{~V} \sim 1500 \mathrm{~V}$ \\
Active dimensions & $28 \mathrm{~mm} \times 14 \mathrm{~mm}$ \\
Overall dimension & $38 \mathrm{~mm} \times 20 \mathrm{~mm}$ \\
Capacitance & $0.61 \mathrm{ppm}$ \\
Free strain & $1550 \mathrm{ppm}$ \\
Thickness & $300 \mu \mathrm{m}$ \\
Actuator bandwidth & $0 \mathrm{~Hz} \sim 700 \mathrm{KHz}$ \\
Sensor bandwidth & $0 \mathrm{~Hz} \sim 1 \mathrm{MHz}$ \\
\hline
\end{tabular}

of bonding condition of the MFC patch on the Lamb wave signal was also investigated, including the poorly adhered and the correctly adhered bonding conditions and the use of two kinds of couplants (longitudinal wave couplant and shear wave couplant). In order to illustrate the performance of defect detection, an artificial defect was fabricated on the surface of the specimen which was inspected in the pitchcatch mode.

\section{MFC Transducer}

The typical type of MFC transducer tested in this paper is an M2814-P1 (Smart Material Corp.), which is shown in Figure 1. This type of MFC, which is available in $\mathrm{d} 33$ operational mode, actuates and senses along the length of the MFC patch. The specific information is listed in Table 1.

\section{Experiments and Results}

3.1. Dispersion Curves. In our study, a $2 \mathrm{~mm}$ thick aluminum alloy plate was used as specimen. Initially, the theoretical dispersion curves of the relevant Lamb waves were calculated. These are presented, in terms of phase velocity and group velocity, in Figures 2(a) and 2(b). Si and Ai indicate the symmetric and antisymmetric modes $(i=0,1,2 \ldots)$, respectively. They will be used for mode identification and to predict the time of flight of a specific mode.

\subsection{Excitation and Detection of the Lamb Wave Using MFC} Patches. Figure 3 shows the experimental set-up to demonstrate the performance of MFC patches to excite and detect the Lamb waves. A pair of MFC patches was bonded on the surface of the plate; one MFC patch was used as an actuator to generate the Lamb waves and the other was used as a

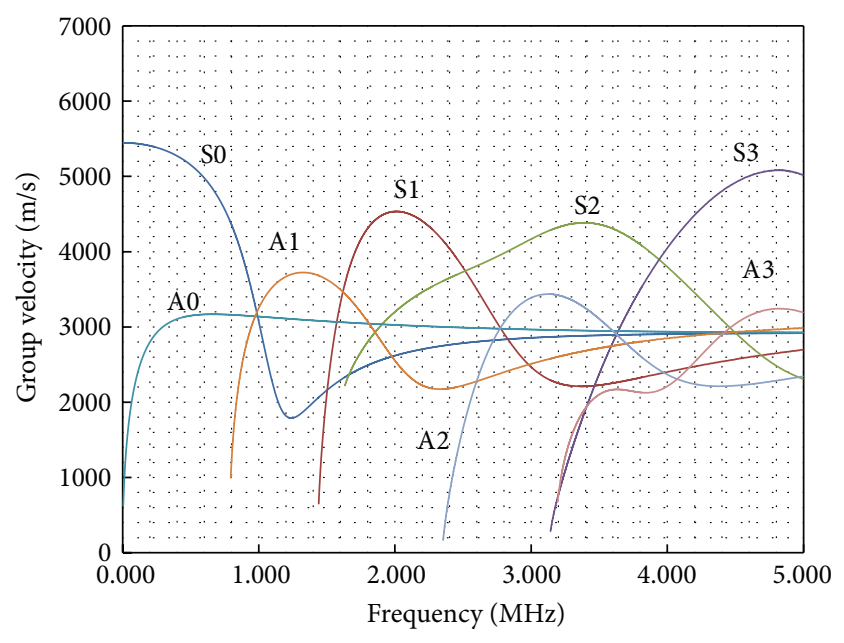

(a)

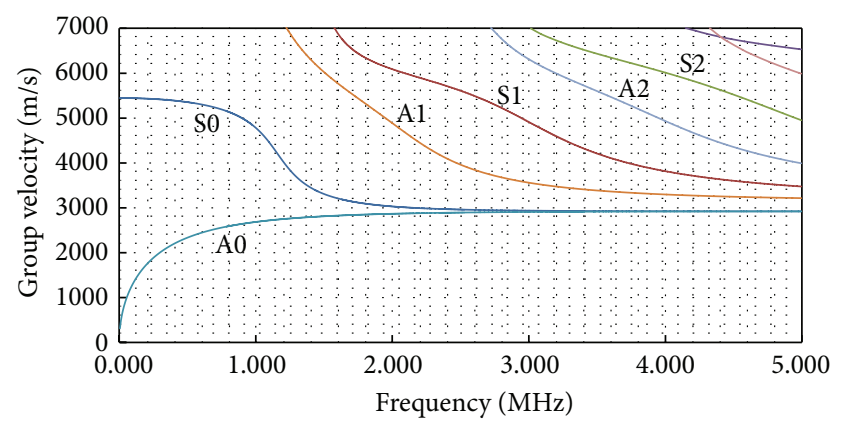

(b)

FIgURE 2: (a) Group velocity dispersion curves. (b) Phase velocity dispersion curves.

sensor to receive the wave signal. A high power tone-burst signal generator (Ritec RAM 5000, USA) was used to drive the actuator. A pulser receiver (Panametrics PR500, USA), which was connected to the sensor, was used to receive the wave signal for the pitch-catch mode inspection.

In order to determine which frequency range was suitable for the embedded MFC patches to generate and detect the Lamb waves, the input signal frequency was varied from $0.1 \mathrm{MHz}$ to $0.6 \mathrm{MHz}$ at $0.05 \mathrm{MHz}$ intervals. This frequency range was chosen because the upper frequency limit of the MFC actuator was $0.7 \mathrm{MHz}$. From the experimental signals shown in Figure 4, it is apparent that the S0 mode is readily detectable within the range from $0.25 \mathrm{MHz}$ to $0.45 \mathrm{MHz}$, while A0 mode is detectable between $0.2 \mathrm{MHz}$ and $0.35 \mathrm{MHz}$. From these results, the $0.3 \mathrm{MHz}$ frequency was selected for the input frequency to use for both of S0 and A0 modes.

Figure 5 shows an example of the detected signal in a time domain, and we can see that the S0 and A0 modes are clearly observed. This signal was short-time-Fourier transformed and overlapped on the theoretical group velocity dispersion curves to verify the Lamb wave modes. The experimental results agree closely with the theoretical dispersion curves at $0.3 \mathrm{MHz}$ for both modes. 


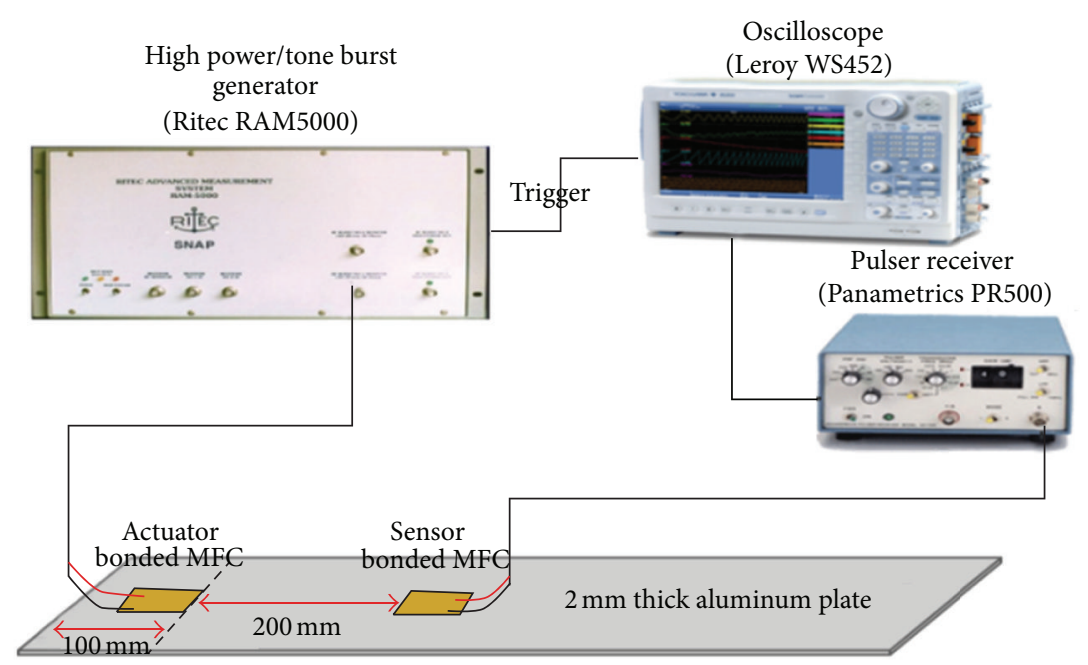

FIgURE 3: Pitch-catch measurement experiment set-up.

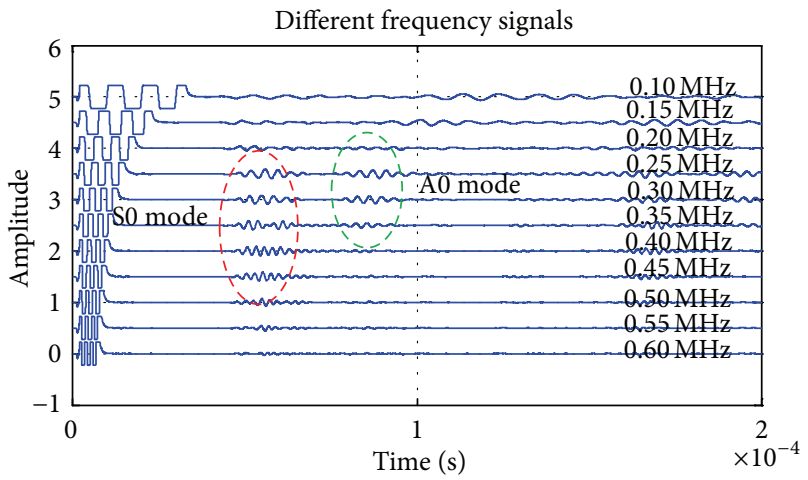

FIgURE 4: Received signals from various frequencies at $0.05 \mathrm{MHz}$ intervals.

3.3. Influence of the Bonding Condition. As the bonding condition of MFC patch may affect the effectiveness of the actuation and sensitivity of the sensing, the quality of the bond is an important consideration in the performance of the system [12]. In order to investigate the influence of the bond quality, we tested several different bonding conditions: couplant-bonded, poorly adhered, and correctly adhered. For the correctly adhered condition, an epoxy adhesive $(3 \mathrm{M}$ Scotch-Weld) was used, which is composed of two parts. The poorly adhered condition was obtained by using only one part of the epoxy, causing degradation in the shear and longitudinal stiffness compared to that of the mixed two-part epoxy adhesive [13].

Figure 6 shows the envelope of detected signals for each bonding condition, where the envelope of signal was obtained by using the Hilbert transform. We can see that the sensor with the correctly adhered condition produced a significantly larger output than those with poorer bonding, and thus proper bonding is very important to ensure the maximum sensitivity of the MFC patch.

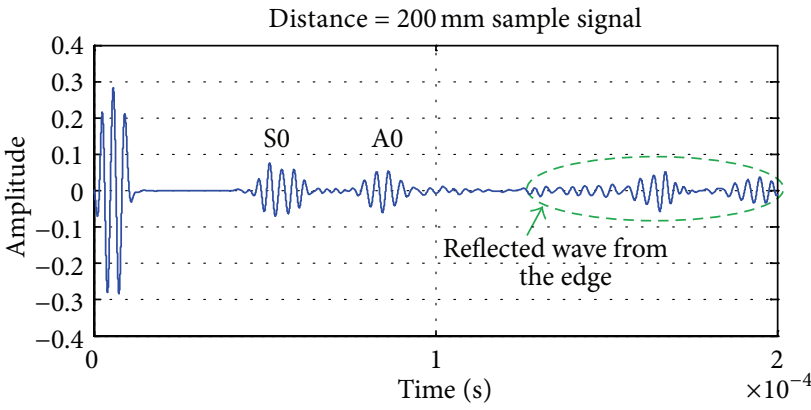

(a)

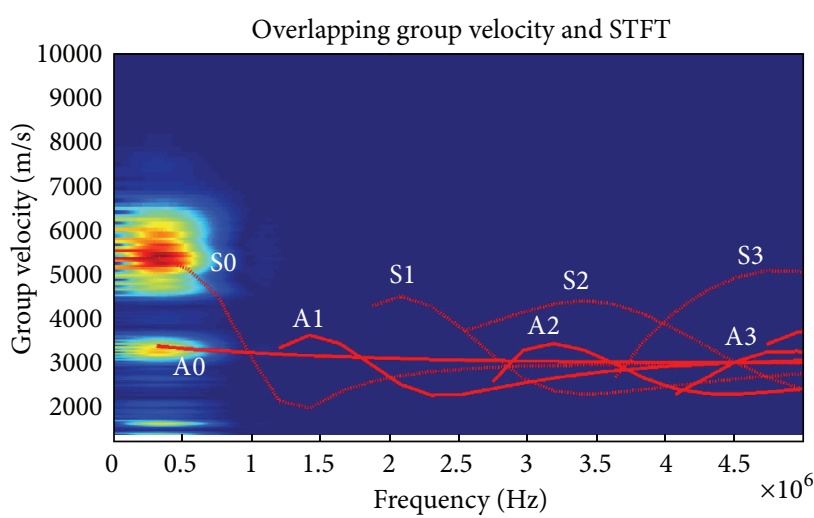

(b)

FIGURE 5: (a) The signal generated and received by MFC patches. (b) Overlapping the group velocity with STFT.

3.4. Defect Detection. In order to further verify the performance of an MFC Lamb wave actuator sensor system for defect detection, an artificial defect on the surface of the specimen was inspected. The defect was a circular groove with diameter of $20 \mathrm{~mm}$, as shown in Figure 7. We used the pitch-catch method. 


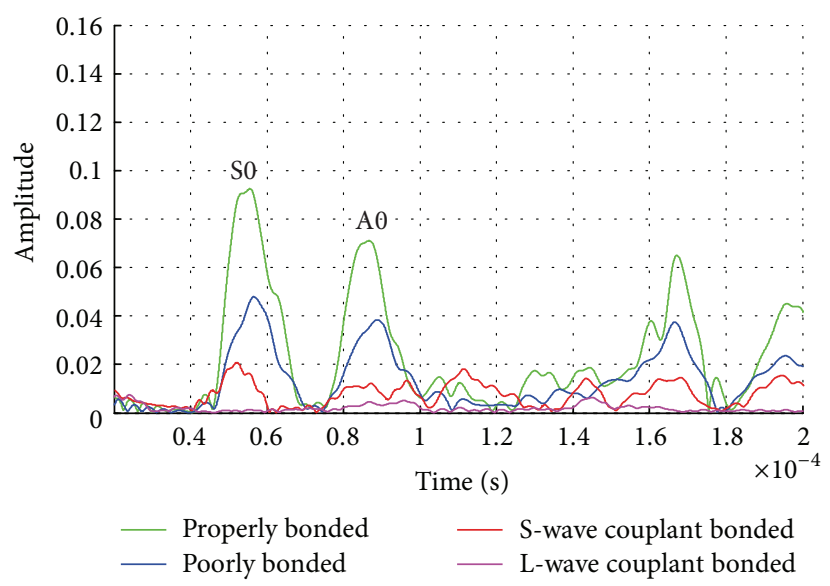

FIGURE 6: The envelope of signals obtained from different bonding conditions.

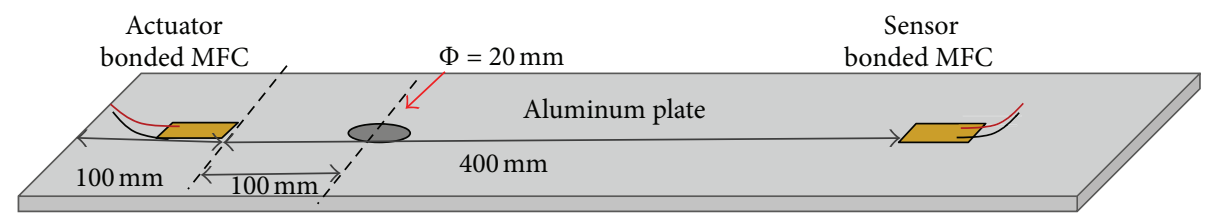

Figure 7: The specimen with circular defect and bonded MFC patches locations.

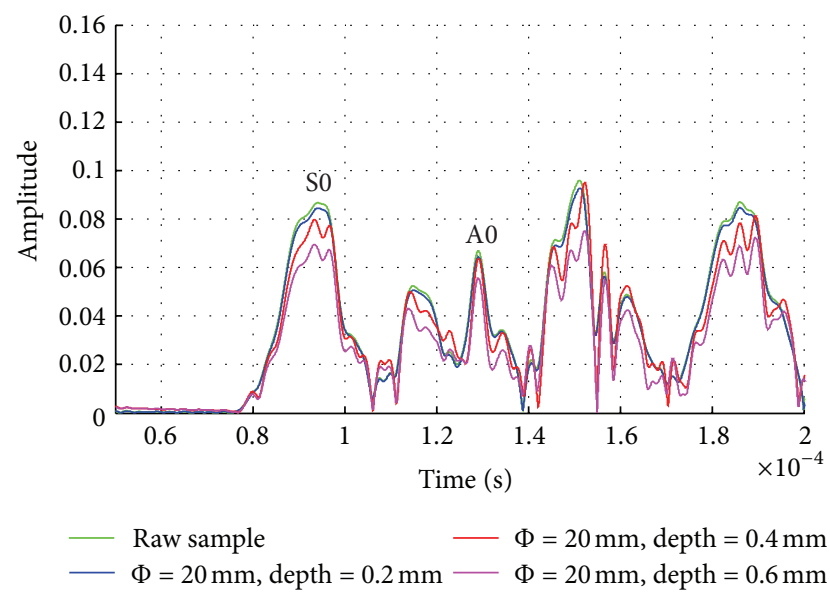

FIGURE 8: Envelope of each signal obtained from each case.

The pitch-catch method can be used to inspect structural degradation that occurs between the actuator and sensor. The main performance of the detection is to compare the Lamb wave's amplitude and time of flight with a pristine case. Due to the dispersive characteristics of the Lamb waves, which are easily influenced by the small changes in the thickness of plate, this method is suitable to detect variations in thickness.

Three different depths of defects, $0.2 \mathrm{~mm}, 0.4 \mathrm{~mm}$, and $0.6 \mathrm{~mm}$, were tested. In order for an easy comparison of the signal amplitude with the baseline, we processed the signals by using the Hilbert transform, which provides the envelope of signal. Figure 8 shows the results. With an increasing of the defect depth, the Lamb wave amplitude decreased consistently. When the defect depth was $0.2 \mathrm{~mm}$, which was $10 \%$ of the plate thickness, the amplitude reduction was relatively small. However, when the defect depth was $20 \%$ and $30 \%$ of the plate thickness, the amplitude was substantially reduced.

\section{Conclusions}

The feasibility of MFC patch transducers for structural health monitoring using the Lamb wave was experimentally demonstrated. For the experiment, a pair of MFC transducers was bonded on a $2 \mathrm{~mm}$ thick aluminum plate specimen. The Lamb waves were well generated and received by this pair of transducers, and the Lamb wave modes were identified by comparing with the theoretical dispersion curves. The bonding quality affected the actuation and the sensitivity of the sensing, so correct bonding is required for the MFC patch to be used for an effective application. Lamb wave inspection, using MFC patch transducer in pitch-catch mode, was also useful to detect the defect of thickness reduction. A $20 \%$ reduction of thickness was detectable. From these results, we can conclude that MFC patch transducers can be effectively used for structural health monitoring using the Lamb waves.

\section{Acknowledgment}

This work was financially supported by the National Research Foundation of Korea (NRF), Grant funded by the Korean government (NRF-2013M2A2A9043241). 


\section{References}

[1] A. B. Thein, G. Park, and C. R. Farrar, "Health monitoring of pipeline systems using macro-fiber composite active-sensor," Steel Structures, vol. 7, pp. 33-48, 2007.

[2] S. B. Kim and H. Sohn, "Instantaneous reference-free crack detection based on polarization characteristics of piezoelectric materials," Smart Materials and Structures, vol. 16, no. 6, pp. 2375-2387, 2007.

[3] V. Giurgiutiu, "Tuned Lamb wave excitation and detection with piezoelectric wafer active sensors for structural health monitoring," Journal of Intelligent Material Systems and Structures, vol. 16, no. 4, pp. 291-305, 2005.

[4] H. A. Sodano, G. Park, and D. J. Inman, "An investigation into the performance of macro-fiber composites for sensing and structural vibration applications," Mechanical Systems and Signal Processing, vol. 18, no. 3, pp. 683-697, 2004.

[5] M. Barbezat, A. J. Brunner, P. Flueler, C. Huber, and X. Kornmann, "Acoustic emission sensor properties of active fibre composite elements compared with commercial acoustic emission sensors," Sensors and Actuators A, vol. 114, no. 1, pp. 13-20, 2004.

[6] http://www.smartmaterials.com/.

[7] Q. Q. Zhang, S. J. Gross, S. Tadigadapa, T. N. Jackson, F. T. Djuth, and S. Trolier-McKinstry, "Lead zirconate titanate films for d33 mode cantilever actuators," Sensors and Actuators A, vol. 105, no. 1, pp. 91-97, 2003.

[8] X. Lin and F. G. Yuan, "Diagnostic Lamb waves in an integrated piezoelectric sensor/actuator plate: analytical and experimental studies," Smart Materials and Structures, vol. 10, no. 5, pp. 907913, 2001.

[9] R. B. Williams, G. Park, D. J. Inman, and W. K. Wilkie, "An overview of composite actuators with piezoceramic fibers," in Proceedings of the 20th International Modal Analysis Conference, pp. 421-427, Los Angeles, Calif, USA, February 2002.

[10] H. M. Matt and F. L. di Scalea, "Macro-fiber composite piezoelectric rosettes for acoustic source location in complex structures," Smart Materials and Structures, vol. 16, no. 4, article 064, pp. 1489-1499, 2007.

[11] A. B. Thien, H. C. Chiamori, J. T. Ching, J. R. Wait, and G. Park, "The use of macro-fibre composites for pipeline structural health assessment," Structural Control and Health Monitoring, vol. 15, no. 1, pp. 43-63, 2008.

[12] F. L. di Scalea, H. Matt, I. Bartoli, S. Coccia, G. Park, and C. Farrar, "Health monitoring of UAV wing skin-to-spar joints using guided waves and macro fiber composite transducers," Journal of Intelligent Material Systems and Structures, vol. 18, no. 4, pp. 373-388, 2007.

[13] http://solutions.3m.com/wps/portal/3M/en_US/Adhesives/ Tapes/Products/ /3M-Tape-and-3M-Adhesives/StructuralAdhesives? $\mathrm{N}=5024768 \& \mathrm{rt}=\mathrm{c} 3$. 

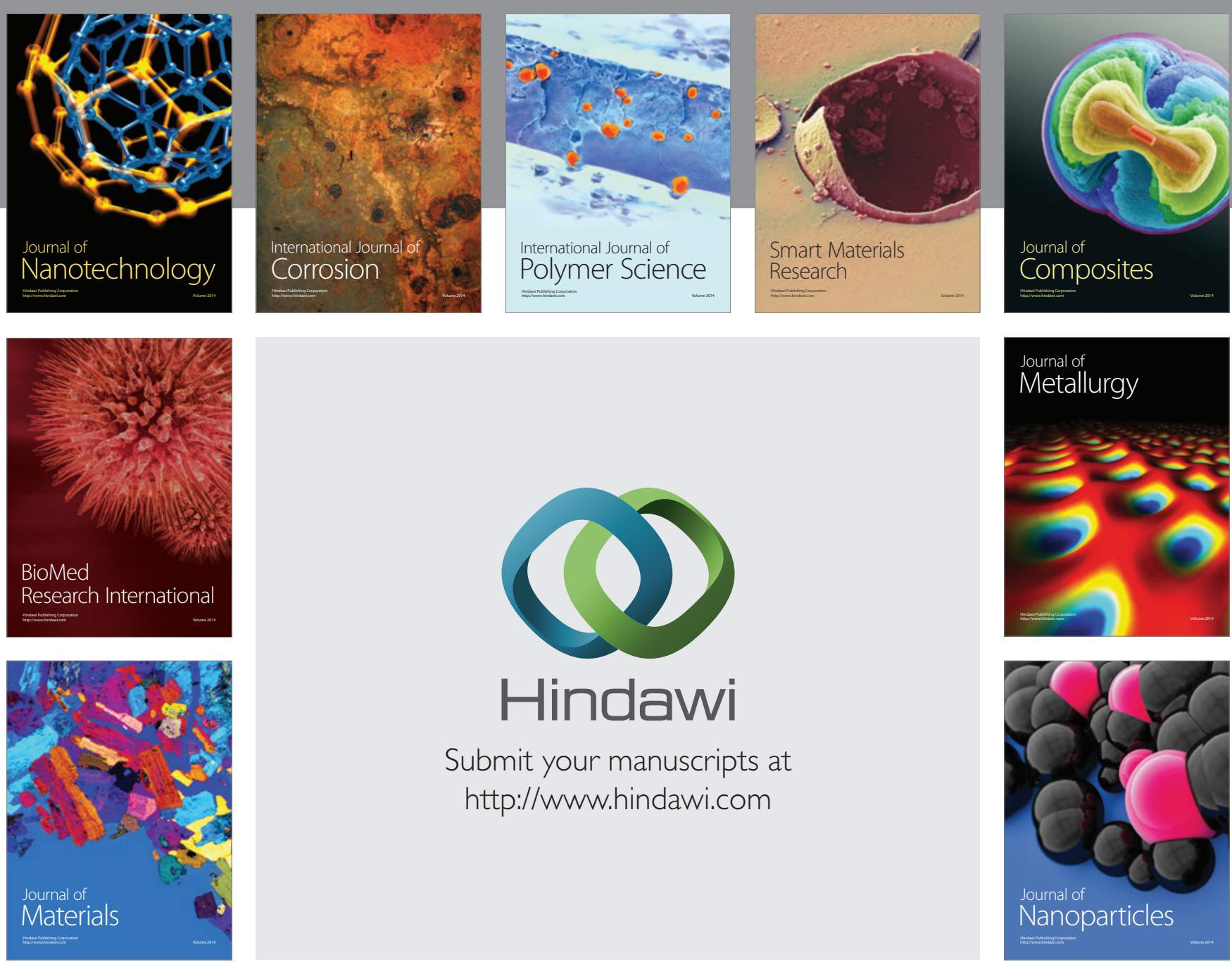

Submit your manuscripts at http://www.hindawi.com
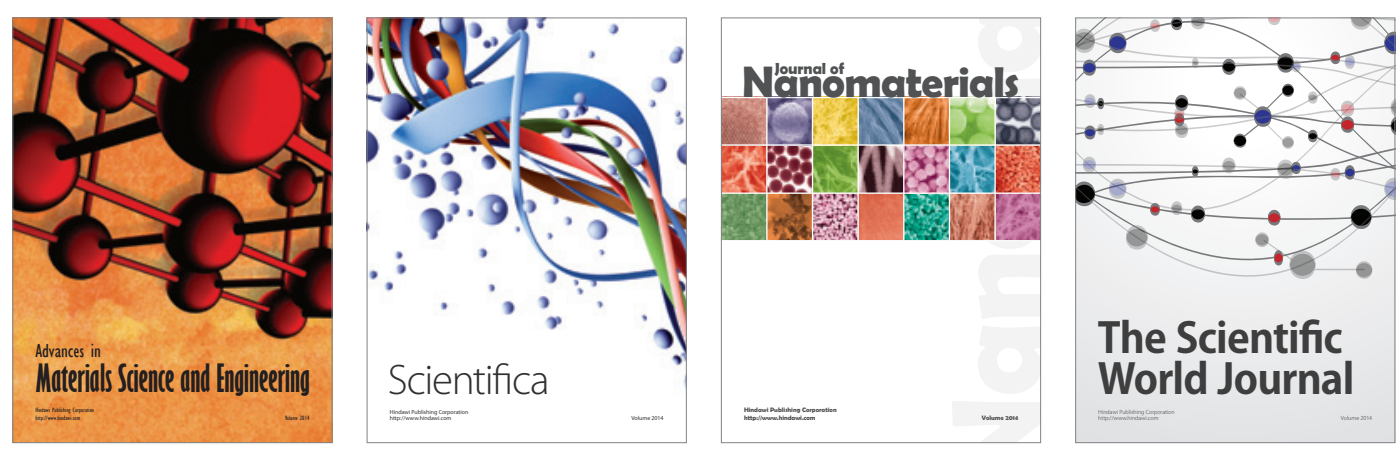

\section{The Scientific World Journal}
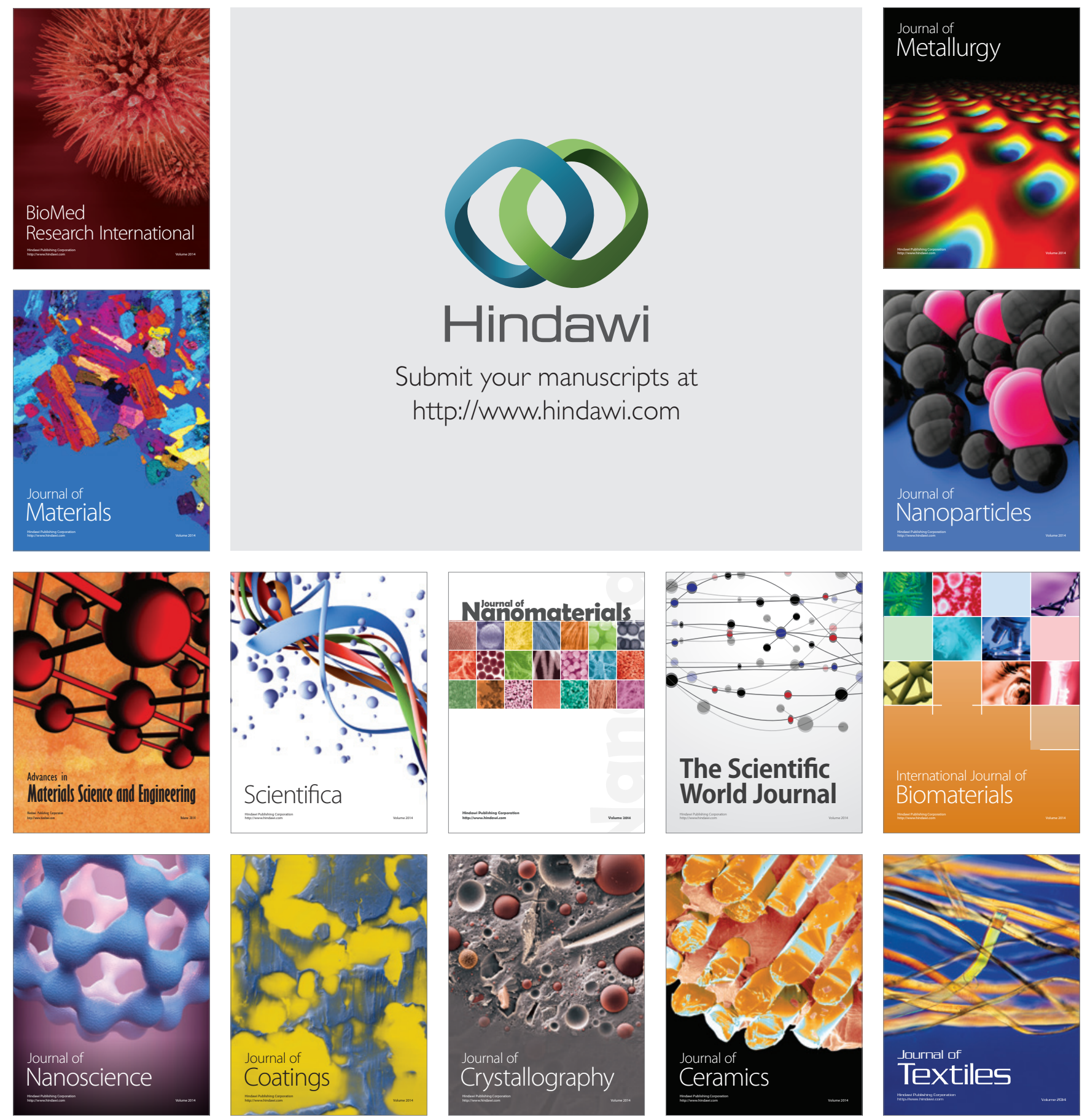